\title{
Absence of Intercellular Antigens in the Deep Layers of the Epidermis in Pemphigus Foliaceus
}

\author{
Jean-Claude Bystryn and Jose Rodriguez, Department of Dermatology, \\ New York University School of Medicine, New York 10016
}

\begin{abstract}
A B S T R A C T 12 patients with pemphigus foliaceus, a form of pemphigus with lesions that arise in the intercellular substance in the superficial layers of the epidermis, and 7 patients with pemphigus vulgaris, where lesions are in the deep layers, were studied by immunofluorescence. Circulating antibodies to intercellular antigens (IC antibodies) were found in 11 pemphigus foliaceus and 5 pemphigus vulgaris patients. On direct immunofluorescence of skin lesions $75 \%$ (9 of 12), pemphigus foliaceus patients had intercellular deposits of IgG localized solely or predominantly in the superficial epidermal layers, whereas this was not the case in any of the patients with pemphigus vulgaris. Over $70 \%$ of the pemphigus foliaceus patients with predominantly superficial IgG deposits lacked in their lesions normal intercellular antigens usually expressed in the deep layers of the epidermis. This was shown by the inability of IC antibodies in autologous or allogeneic sera to bind to intercellular antigens in the lower epidermis of patient's skin, even though the same sera could bind to intercellular antigens in all layers of normal allogeneic skin. Lack of normal intercellular antigens deep in the epidermis may result in circulating IC antibodies binding to the superficial layers, a site which corresponds to, and thus in some patients may account for, the anatomical location of lesions in pemphigus foliaceus.
\end{abstract}

\section{INTRODUCTION}

Pemphigus is an uncommon bullous eruption of the skin characterized by recurrent painful blisters and erosions in the oral cavity and skin. Untreated, the disease is usually fatal. There are several forms of pemphigus which are differentiated principally by the level of lesions in the epidermis. The most common form of pemphigus, pemphigus vulgaris, is characterized by bullae which arise deep in the epidermis just

Received for publication 29 April 1977 and in revised form 26 September 1.977. above the basal cell layer. In pemphigus foliaceus, in contrast, the lesions arise in the superficial epidermis. In all forms of pemphigus, the site of the earliest defect visualizable by electron microscopy is in the intercellu$\operatorname{lar}(\mathrm{IC})^{1}$ substance $(1,2)$.

Pemphigus is associated with a unique immunologic abnormality. More than $80 \%$ of patients with this disease develop intercellular antibodies (3-9) to antigens which are normal constituents of the intercellular substance in human and mammalian epidermis $(10,11)$. Immunoglobulins $(4,12-14)$ together with complement $(13,15)$ are deposited in vivo in the intercellular substance of lesions and normal skin of patients with pemphigus. The correlation between the anatomical position of intercellular antigens and the earliest histological lesions of pemphigus, and between the titer of IC antibodies and the severity of the disease $(5,16-18)$, suggests that IC antibodies play a role in the pathogenesis of the disease. The production of intraepidermal acantholytic bullae similar to those of pemphigus by IC antibodies incubated with human epidermis in organ culture (19) or repeatedly injected into the skin (20) or oral mucosa (21) of animals supports this concept.

The reasons for the development of bullae in different layers of the epidermis in the various forms of pemphigus are unknown. However, it has been found that some patients with pemphigus foliaceus have IC antibodies to antigens present only in the superficial epidermal layers (22), in contrast to the antibodies in other forms of pemphigus which react to intercellular antigens throughout the epidermis. This unusual type of IC antibodies (subcorneal IC antibodies) has not been found in patients with other forms of pemphigus or other bullous diseases and may provide one explanation for the superficial location of acantholysis in pemphigus foliaceus.

We now report the association of another immunologic

${ }^{1}$ Abbreviations used in this paper: IC, intercellular; PBS, phosphate-buffered saline. 
abnormality with pemphigus foliaceus, namely the absence in some patients of intercellular antigens normally expressed in the lower layers of the epidermis. As a consequence, IC antibodies may only be able to bind to the superficial epidermis, a site which corresponds to $(2,23)$, and may in some patients account for, the anatomical location of lesions of pemphigus foliaceus.

\section{METHODS}

Patients. All patients in this study had typical pemphigus vulgaris or pemphigus foliaceus by both clinical and histological criteria. Sera and tissue specimens were collected from all patients during the active phase of their disease and stored at $-30^{\circ} \mathrm{C}$. Specimens of involved and normal skin were obtained by punch biopsy and quick-frozen in liquid nitrogen. In one patient, skin specimens were also obtained during remission of the disease. Normal human skin obtained from surgical specimens and monkey and guinea pig esophagus were used for control studies. Tissue specimens were stored for no longer than $1 \mathrm{wk}$ and before use were cut into $4 \mu \mathrm{m}$ sections with a cryostat.

Antisera. Fluorescein-labeled conjugates to human IgG, IgM, IgA, and C3 were purchased from Hyland Div., Travenol Laboratories, Inc. (Costa Mesa, Calif.). All sera were tested for specificity by immunoelectrophoresis. The total protein concentrations of the conjugates were, respectively, 10.3, 20.4, 20.5 , and $10.1 \mathrm{mg} / \mathrm{ml}$; the specific antibody concentration 2.0 , $1.9,1.9$, and $1.6 \mathrm{mg} / \mathrm{ml}$; the specific antibody protein-tofluorescein molar ratio $0.066,0.028,0.041$, and 0.07 ; and the fluorescein-to-protein molar ratio $3.0,3.3,2.3$, and 2.3. For use, the conjugates were diluted $1 / 32,1 / 32,1 / 32$, and $1 / 64$, respectively, in phosphate-buffered saline (PBS), pH 7.3, with $4 \%$ bovine serum albumin (Schwartz Mann Div., Becton, Dickinson \& Co., Orangeburg, N.Y.). These dilutions were determined by chessboard titrations (11).

Immunofluorescence studies. Indirect immunofluorescence was used to test sera for IC antibodies. The tests were performed by a standard technique (11), as previously described (24). Briefly, $0.2 \mathrm{ml}$ of the appropriate dilution of human serum in PBS was incubated for $30 \mathrm{~min}$ at $27^{\circ} \mathrm{C}$ with cryostat-cut sections of frozen normal allogeneic skin and monkey and guinea pig esophagus. After washing for $10 \mathrm{~min}$ in PBS, the specimens were reincubated with $0.2 \mathrm{ml}$ of the appropriate dilution of fluorescein-labeled conjugate for 30 min at $27^{\circ} \mathrm{C}$, washed for $45 \mathrm{~min}$ in PBS, and covered with $0.2 \mathrm{ml}$ of mounting medium ( $90 \%$ glycerol in PBS). The slides were examined with a binocular microscope equipped with a mercury vapor lamp and FITC and No. 50 barrier filters. Direct immunofluorescence tests for in vivo deposits of Ig and C3 were performed by established methods (11).

The presence of intercellular antigens in skin specimens was determined by indirect immunofluorescence. For this purpose, the specimens were reacted with pemphigus vulgaris sera known to have antibodies to intercellular antigens in all epidermal layers. The titer of IC antibodies in these sera ranged from 320 to above 1,280 . For use, all sera were diluted $1 / 20$ in PBS.

In vitro complement binding. The ability of antibodies in pemphigus sera to fix complement when bound to intercellular antigens in human skin was tested by a complement binding technique described by Jordon et al. (25). Briefly, undiluted and 1/5 dilution of patients' sera in PBS were incubated for 30 min at $27^{\circ} \mathrm{C}$ on cryostat-cut sections of frozen normal allogeneic skin. Each section was washed five times with PBS and incubated for $30 \mathrm{~min}$ with a $1 / 10$ dilution of fresh normal human serum in PBS as a complement source. The sections were washed five times in PBS and incubated with fluorescein-labeled conjugate to human C3 for $30 \mathrm{~min}$. Thereafter, the slides were washed and read as described above for immunofluorescence studies.

\section{RESULTS}

Indirect immunofluorescence. During the acute phase of their illness, 11 of the 12 patients with pemphigus foliaceus had in their sera IC antibodies to monkey and (or) guinea pig esophagus. The titers ranged from 80 to above 1,280 (Table I). Subcorneal IC antibodies, reacting solely to superficial intercellular antigens in guinea pig esophagus, were present in one patient to a titer of 160 .

Direct immunofluorescence. Direct immunofluorescence studies were performed on skin lesions of 12 patients with pemphigus foliaceus. Intercellular IgG deposits were present in all but one. This patient did not have circulating IC antibodies. As can be seen in Table II, the deposits were predomi-

TABLE I

Direct Immunofluorescence Studies of Skin for Location of Intercellular Deposits of IgG Lesions

\begin{tabular}{|c|c|c|c|}
\hline \multirow[b]{2}{*}{ Diagnosis } & \multirow{2}{*}{$\begin{array}{c}\text { Titer* } \\
\text { IC antibodies }\end{array}$} & \multicolumn{2}{|c|}{$\begin{array}{c}\text { Location of } \\
\text { intercellular } \\
\text { IgG deposits }\end{array}$} \\
\hline & & Superficial & Deep \\
\hline \multicolumn{4}{|c|}{ Pemphigus foliaceus } \\
\hline 2844 & $>1280$ & ++ & - \\
\hline 3680 & $>1280$ & ++ & - \\
\hline 3842 & $>1280$ & +++ & \pm \\
\hline 3895 & 0 & - & - \\
\hline 4009 & 320 & + & + \\
\hline 4137 & $160 \S$ & ++ & - \\
\hline 4266 & 80 & - & ++ \\
\hline 4306 & 160 & ++ & - \\
\hline 4326 & 320 & ++ & - \\
\hline 4406 & $>1280$ & ++ & + \\
\hline 4443 & $>1280$ & ++ & - \\
\hline 4424 & 0 & ++ & \pm \\
\hline \multicolumn{4}{|c|}{ Pemphigus vulgaris } \\
\hline 1830 & 320 & - & ++ \\
\hline 3468 & 640 & - & ++ \\
\hline 3773 & 80 & ++ & ++ \\
\hline 3844 & 80 & + & + \\
\hline 3864 & 80 & ++ & $+t$ \\
\hline 4075 & 0 & - & - \\
\hline 4308 & 640 & ++ & ++ \\
\hline
\end{tabular}

* Reciprocal of highest dilution giving + reaction by indirect immunofluorescence.

$\ddagger$ By direct immunofluorescence.

$\S$ Subcorneal intercellular antibodies. 
TABLE II

Direct Immunofluorescence Studies of Skin for Location of Intercellular Deposits of C3 Lesions

\begin{tabular}{|c|c|c|}
\hline \multirow[b]{2}{*}{ Diagnosis } & \multicolumn{2}{|c|}{$\begin{array}{l}\text { Location of intercellular } \\
\text { C3 deposits* }\end{array}$} \\
\hline & Superficial & Deep \\
\hline \multicolumn{3}{|c|}{ Pemphigus foliaceus } \\
\hline 2844 & ++ & - \\
\hline 3680 & - & + \\
\hline 3842 & + & ++ \\
\hline 3895 & - & - \\
\hline 4009 & + & ++ \\
\hline 4137 & - & - \\
\hline 4266 & - & ++ \\
\hline 4306 & - & ++ \\
\hline 4326 & ++ & + \\
\hline 4406 & - & ++ \\
\hline 4443 & - & ++ \\
\hline 4424 & - & ++ \\
\hline \multicolumn{3}{|c|}{ Pemphigus vulgaris } \\
\hline 1830 & - & ++ \\
\hline 3468 & - & - \\
\hline 3773 & - & ++ \\
\hline 3844 & - & - \\
\hline 3864 & + & ++ \\
\hline 4075 & - & - \\
\hline 4308 & ++ & ++ \\
\hline
\end{tabular}

* By direct immunofluorescence.

nantly in superficial epidermal layers (Fig. 1A). They were present only in this location in six patients and were heaviest in this area in an additional three. Thus, in $75 \%$ ( 9 of 12) of patients with pemphigus foliaceus, in vivo intercellular deposits of IgG were solely or predominantly superficial. By contrast, this was not the case in patients with pemphigus vulgaris. Intercellular IgG deposits were present in lesions of six of seven patients with this form of pemphigus. In two patients the deposits were only in the deep epidermis, and in the other four they were present throughout the epidermis (see Fig. 2). Intercellular IgM deposits were found in one pemphigus foliaceus patient and in none of those with pemphigus vulgaris. In this patient, the deposits were superficial. There were no IgA deposits in any patients.

Intercellular deposits of $\mathrm{C} 3$ were present in lesions of 10 of $12(83 \%)$ of the patients with pemphigus foliaceus and in 4 of 7 (57\%) of those with pemphigus vulgaris. In both diseases, the deposits were predominantly in the deep layer of the epidermis. They were exclusively in this area in six of the pemphigus foliaceus patients and heaviest in this area in another two. Thus, in $80 \%$ of the pemphigus foliaceus patients, C3 was deposited predominantly in the deep epidermis. As implicated by these findings, there was no correlation between the site of IgG and C3 deposits.
Three possibilities were considered to account for the superficial localization of intercellular deposits of IgG in pemphigus foliaceus: (a) Circulating IC antibodies in this disease may be of restricted specificity and not directed, and thus unable to bind, to deep intercellular antigens. $(b)$ Some intercellular antigens may not be expressed in the lower epidermis in lesions of pemphigus foliaceus. (c) IgG may be blocked from binding to deep intercellular antigens by other classes of Ig or by complement already deposited at these sites.

Specificity of circulating IC antibodies. The specificity of IC antibodies in the 10 pemphigus foliaceus patients with diminished or absent deposits of IgG in their deep epidermis was studied by reacting their sera at a 1/20 dilution with guinea pig and monkey esophagus and normal allogeneic skin. As can be seen in Table III, only one patient had subcorneal IC antibodies which reacted only to superficial intercellular antigens in guinea pig esophagus. Two patients did not have IC antibodies. The remaining seven patients had IC antibodies reactive to intercellular antigens in all layers of allogeneic skin and zenogeneic esophagus. This finding indicates that restricted specificity of IC antibodies cannot account for the superficial location of in vivo bound IgG in the majority of patients with this phenomenon.

Expression of intercellular antigens in deep epidermal layers of lesions. The presence of intercellular antigens in lesions of six pemphigus foliaceus patients without intercellular deposits of IgG in the lower epidermis and one patient with very faint deposits was studied as described in Methods. Allogeneic IC antibodies in pemphigus vulgaris sera were unable to bind to deep epidermal intercellular antigens in five of the seven $(71 \%)$ patients (see Table IV). Autologous IC antibodies were unable to bind to the lower epidermis in four of five $(80 \%)$ patients (Figs. $1 \mathrm{~B}$ and $3 \mathrm{~A}$ ). The remaining two patients were not studied because they lacked IC antibodies. One patient (3842) lacked deep intercellular antigens reactive to autologous but not to allogeneic IC antibodies (see Figs. 3A-C) whereas the converse was true in another patient (4306). In all cases, IC antibodies in pemphigus foliaceus and pemphigus vulgaris sera were able to bind to intercellular antigens in the lower layers of normal allogeneic epidermis (see Tables III and IV, and Figs. IC and 3C), indicating that the lack of binding to pemphigus foliaceus lesions was due to the absence of appropriate antigens in this area. Overall, deep intercellular antigens reactive to autologous sera were not expressed in lesions of $33 \%$ of pemphigus foliaceus patients and in $80 \%$ of those who did not have in vivo intercellular deposits of $\operatorname{IgG}$ in this location. In a somewhat smaller proportion of patients, IC antigens reactive to allogeneic antibodies were also absent. 


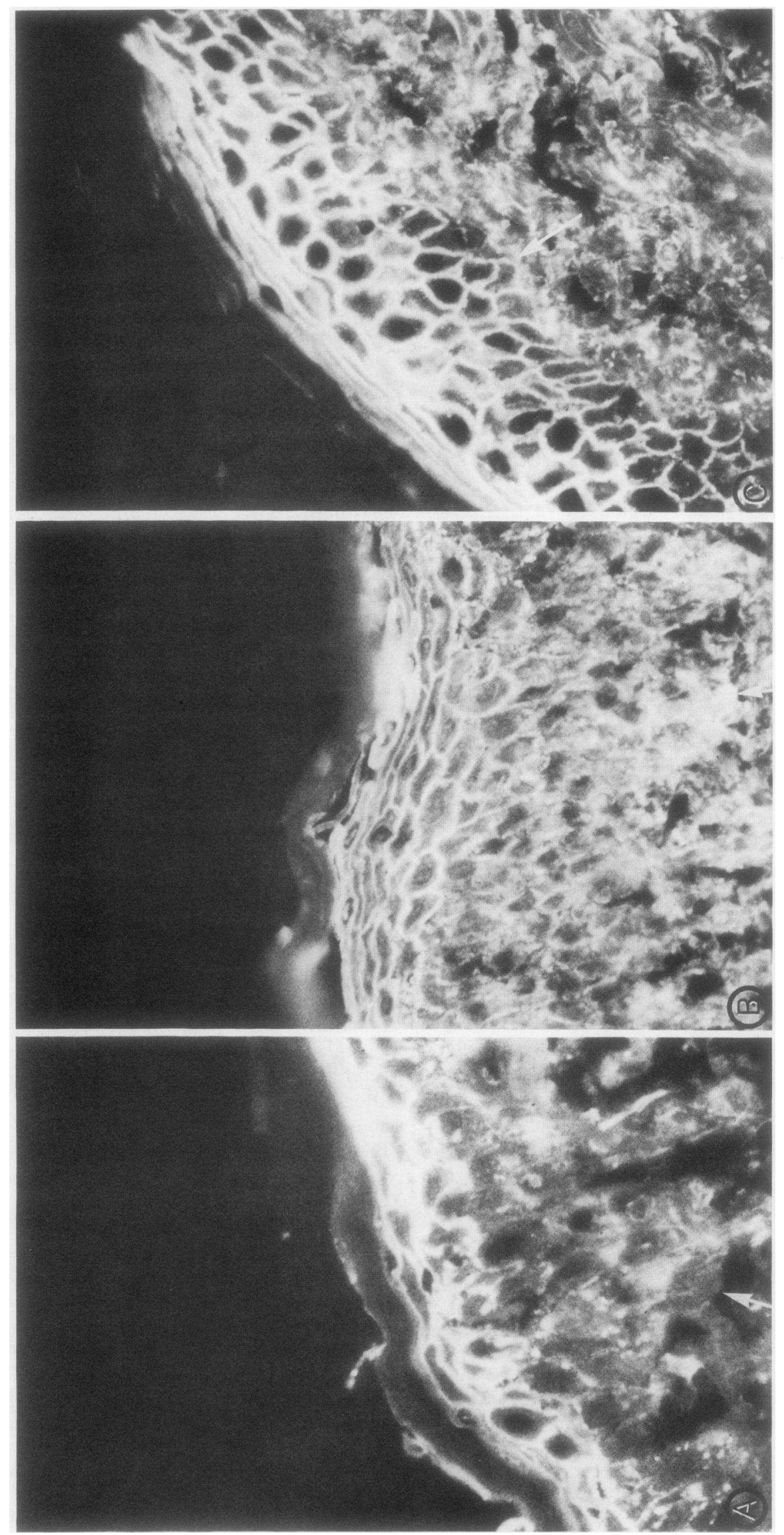






FIgURE 2 Patient 3773. Direct immunofluorescence of pemphigus vulgaris lesion. Intercellular deposits of IgG are present in all layers of the epidermis. $\times 228$.

Effect of in vivo deposits of Ig or complement on binding of intercellular antibodies. The possibility that Ig or complement bound in vivo to intercellular antigens in the deep epidermis blocked the binding of other IC antibodies was unlikely because of the following observations: (a) There were no in vivo deposits of IgG, IgM, or IgA in the deep epidermal layers of the five patients lacking IC antigens in this
TABLE III

Specificity of IC Antibodies in Patients with Pemphigus Foliaceus and Absent or Diminished Deep Intercellular Deposits of IgG

\begin{tabular}{|c|c|c|c|c|c|c|}
\hline \multirow{4}{*}{$\begin{array}{l}\text { Pemphigus } \\
\text { foliaceus } \\
\text { sera }\end{array}$} & \multicolumn{6}{|c|}{ Specificity of IC antibodies } \\
\hline & \multicolumn{2}{|c|}{ Esophagus } & \multirow{2}{*}{\multicolumn{4}{|c|}{ Allogeneic normal skin }} \\
\hline & & & & & & \\
\hline & pig & Monkey & 4453 & 4437 & 4528 & 4546 \\
\hline 2844 & $\mathrm{G}$ & G & $\mathrm{G}^{*}$ & G & G & $\mathrm{G}^{*}$ \\
\hline 3680 & G & G & G & $\mathrm{G}$ & G & G \\
\hline 3842 & G & G & G & $\mathrm{G}^{*}$ & G & $\mathrm{G}^{*}$ \\
\hline 3895 & - & - & - & - & - & - \\
\hline 4137 & $\mathrm{U}$ & - & - & - & - & - \\
\hline 4306 & G & G & G & G & G & G \\
\hline 4326 & G & G & $\mathrm{G}$ & NS & G & G \\
\hline 4406 & G & $\mathrm{G}$ & $\mathrm{G}$ & $\mathrm{U}$ & G & G \\
\hline 4443 & G & G & NS & G & G & G \\
\hline 4424 & - & - & - & - & - & NS \\
\hline
\end{tabular}

NS, not studied; U, antibodies directed to IC antigens in upper layer of epidermis only; L, antibodies directed to IC antigens in lower layer of epidermis only; G, antibodies directed to IC antigens in all layers.

* Reactive predominately to U-IC.

location. $\operatorname{IgE}$ and $\operatorname{IgD}$ were not tested for, since their concentration in serum is very low and no abnormal deposits of these classes have been reported in pemphigus. ( $b$ ) There were no deep in vivo deposits of C3 in two of these patients (2844 and 4137), indicating that in at least these cases, blocking by complement was not a factor. (c) In the two patients (3842 and 4306) in which it could be tested, in vivo deposits of C3 in the deep epidermis did not prevent the subsequent in vitro binding of antibodies to IC antigens in this location.

Expression of deep intercellular antigens in uninvolved skin of patients with pemphigus foliaceus. To determine whether the loss of intercellular antigens in pemphigus foliaceus was restricted to lesions or was a more general phenomenon, we examined uninvolved skin in five patients for in vivo deposits of IgG and for the presence of intercellular antigens in the lower epidermis. As can be seen in Table V, intercellular deposits of IgG were present in uninvolved skin in three of five

FIGURE 1 Patient 3680. (A) Direct immunofluorescence of pemphigus foliaceus lesion. Intercellular deposits of $\mathrm{IgG}$ are present only in the superficial layers of the epidermis. There are no deposits in deeper layers. In this and all subsequent figures, the dermal-epidermal junction is indicated by an arrow. (B) Indirect immunofluorescence. Autologous serum reacted with lesion, indicating lack of binding of IC antibodies to deep epidermal layers. Similar results were obtained when the same lesion was reacted with allogeneic pemphigus vulgaris sera. (C) Indirect immunofluorescence. Patient's serum reacted with normal human skin, demonstrating that its IC antibodies can react to intercellular antigens in deep layers of allogeneic epidermis. $\times 217$. 

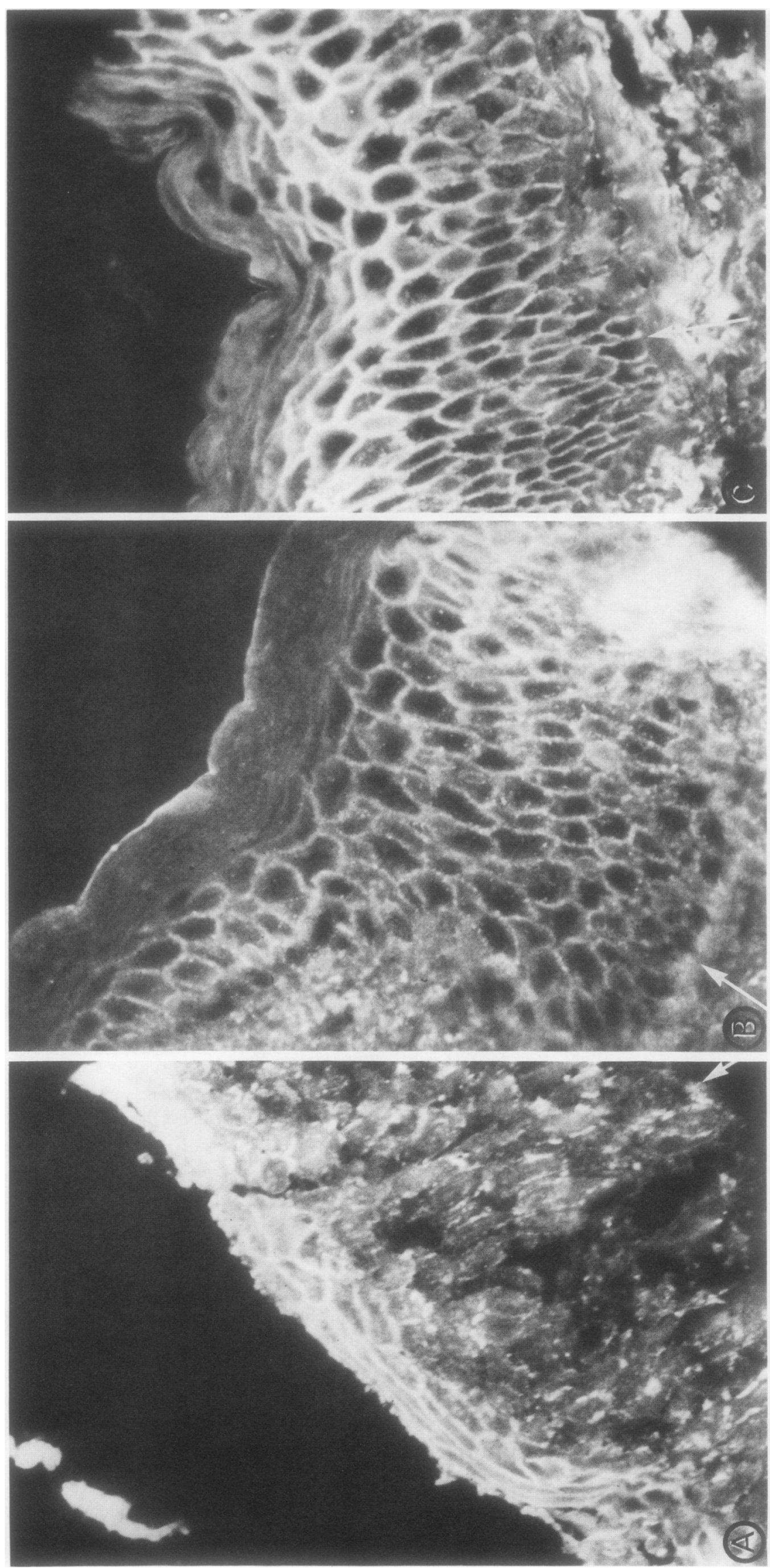
TABLE IV

Presence of Deep Intercellular Antigens in Lesions of Pemphigus Foliaceus and Normal Allogeneic Skin

\begin{tabular}{|c|c|c|c|c|c|c|c|c|c|}
\hline \multirow[b]{3}{*}{ Skin specimen } & \multicolumn{9}{|c|}{ Binding of IC antibodies to deep intercellular antigens* } \\
\hline & \multirow{2}{*}{$\begin{array}{l}\text { Autologous } \\
\text { serum }\end{array}$} & \multicolumn{6}{|c|}{ Pemphigus vulgaris serum } & \multicolumn{2}{|c|}{$\begin{array}{l}\text { Normal } \\
\text { serum }\end{array}$} \\
\hline & & 2103 & 2600 & 2730 & 2832 & 2932 & 3573 & 1 & 2 \\
\hline \multirow{2}{*}{\multicolumn{10}{|c|}{$\begin{array}{l}\text { Pemphigus foliaceus } \\
\text { lesions }\end{array}$}} \\
\hline & & & & & & & & & \\
\hline 2844 & - & & & - & - & & & - & - \\
\hline 3680 & - & - & - & & & & - & - & - \\
\hline 3842 & - & + & + & & & & & - & - \\
\hline $3895 \ddagger$ & NS & + & - & & & & & - & - \\
\hline $4137 \ddagger$ & NS & & & & - & - & & - & - \\
\hline 4306 & + & - & - & & & & - & - & - \\
\hline 4443 & - & & & - & - & - & & - & - \\
\hline \multicolumn{10}{|l|}{ Normal allogeneic } \\
\hline 1 & + & + & + & + & + & + & + & - & - \\
\hline 2 & + & + & + & + & + & + & + & - & - \\
\hline 3 & & + & + & & + & & & & \\
\hline 4 & & + & + & & + & & & & \\
\hline 5 & & + & + & & + & & & & \\
\hline 6 & & + & + & & + & & & & \\
\hline 7 & & + & + & & + & & & & \\
\hline 8 & & + & + & & + & & & & \\
\hline
\end{tabular}

NS, not studied.

* All sera tested at $1 / 20$ dilution.

† Sera of patients 3895 and 4137 were negative for IC antibodies to autologous or allogeneic skin.

patients, but in only one were these exclusively in the superficial layers. Intercellular IgG deposits were present in lesions of four of the five patients, but by contrast, were exclusively localized to the upper epidermis in all cases. The presence of intercellular antigens in the lower epidermis of lesions and uninvolved skin was tested by reacting these tissues with autologous and pemphigus vulgaris sera as previously described. In uninvolved skin, as can be seen in Table VI, deep intercellular antigens reactive to autologous sera in vivo or in vitro or to allogeneic sera in vitro were present in four of five patients. In lesions of the same patients, deep intercellular antigens were present in only one patient and were weakly expressed
TABLE V

Direct Immunofluorescence in Pemphigus Foliaceus Lesions and Uninvolved Skin

\begin{tabular}{ccccc}
\hline & \multicolumn{3}{c}{ Location of intercellular deposits of IgG } \\
\cline { 2 - 5 } $\begin{array}{c}\text { Pemphigus foliaceus } \\
\text { patients }\end{array}$ & Lesion & & \multicolumn{2}{c}{ Uninvolved skin } \\
\cline { 2 - 5 } \cline { 2 - 5 } 2844 & ++ & - & Superficial & Deep \\
3895 & - & - & ++ & - \\
4137 & ++ & - & - & - \\
4306 & ++ & - & - & + \\
4443 & ++ & - & ++ & ++ \\
\hline
\end{tabular}

FIGURE 3 Patient 3842. (A) Indirect immunofluorescence. Autologous serum reacted with patient's lesion, demonstrating absence of deep intercellular antigens reactive to autologous IC antibodies. (B) Indirect immunofluorescence. Pemphigus vulgaris serum reacted with same lesion, demonstrating that tissue retains intercellular antigens in lower epidermis reactive to allogeneic IC antibodies. (C) Indirect immunofluorescence. Patient's serum reacted with normal human skin, demonstrating that its IC antibodies can react to intercellular antigens in lower layers of epidermis. $\times 215$. 
TABLE VI

Presence of Deep Intercellular Antigens in Pemphigus

Foliaceus Lesions and Uninvolved Skin

\begin{tabular}{|c|c|c|c|c|}
\hline \multirow[b]{3}{*}{ Patient } & \multicolumn{4}{|c|}{ Binding of IC antibodies to deep intercellular antigens } \\
\hline & \multicolumn{2}{|c|}{ Lesions } & \multicolumn{2}{|c|}{ Uninvolved skin } \\
\hline & $\begin{array}{l}\text { Autologous } \\
\text { serum }\end{array}$ & $\begin{array}{l}\text { Pemphigus } \\
\text { vulgaris } \\
\text { serum }\end{array}$ & $\begin{array}{c}\text { Autologous } \\
\text { serum }\end{array}$ & $\begin{array}{c}\text { Pemphigus } \\
\text { vulgaris } \\
\text { serum }\end{array}$ \\
\hline 2844 & - & - & - & - \\
\hline $3895^{*}$ & NS & + & NS & + \\
\hline $4137 *$ & NS & - & NS & + \\
\hline 4306 & \pm & - & $+\ddagger$ & NS \\
\hline 4443 & - & - & $+\hbar$ & NS \\
\hline
\end{tabular}

NS, not studied.

* Antibodies to G-IC lacking in autologous sera

$\ddagger$ In vivo.

in a second. These findings indicate that the absence of intercellular antigens in the lower layers of the epidermis in pemphigus foliaceus is predominantly associated with lesions of this disease, though occasionally the process may involve uninvolved skin as well.

Specificity of deep intercellular antigens. The patterns of cross-reactivity observed when pemphigus foliaceus and vulgaris sera were reacted with different specimens of skin indicate that there are several different intercellular antigens in the lower layers of the epidermis. As can be seen in Table III, tissue specimen 4437 lacked deep intercellular antigens reactive with sera 4406 , but had deep intercellular antigens reactive to other sera. In Table IV, lesion 3842 had deep intercellular antigens reactive to allogeneic but not to autologous sera, whereas the reverse was true for lesion 4306. These findings suggest that there may be individual and group-specific intercellular antigens in the lower epidermis, either or both of which may be lost in pemphigus foliaceus.

In vitro complement binding by pemphigus sera. After their interaction with normal allogeneic human skin, none of the pemphigus foliaceus or vulgaris sera were able to fix $\mathrm{C} 3$ in vitro.

\section{DISCUSSION}

This study shows that pemphigus foliaceus may in some instances be associated with a loss of intercellular antigens normally present in the deep layers of the epidermis.

It was found that patients with pemphigus foliaceus had IgG fixed in vivo exclusively or predominantly to the intercellular substance in the uppermost layers of the epidermis. In contrast, in pemphigus vulgaris, IgG was deposited in the deeper layers as well. Several possibilities were entertained to account for this finding. The first was that the specificity of IC antibodies in patients with pemphigus foliaceus was restricted to superficial intercellular antigens. Subcorneal IC antibodies reactive only to superficial antigens occur in a few patients with pemphigus foliaceus (22). In the present study, subcorneal IC antibodies were found in only 1 of 12 patients. The antibodies in the other patients were capable of reacting to intercellular antigens in all layers of normal allogeneic or xenogeneic stratified squamous epithelium, thus excluding this possibility from consideration. The second possibility was that some normal intercellular antigens were not expressed in the lower epidermis of the patients. This appeared to be the case in several patients, as evidenced by the inability of autologous or allogeneic IC antibodies to bind to the intercellular substance in deep layers of their epidermis. This was due to a lack of intercellular antigens normally expressed in this location, since the same sera could bind to deep intercellular antigens in normal allogeneic skin. It was unlikely that $\mathrm{C} 3$ or other classes of immunoglobulin bound in vivo to deep intercellular antigens were preventing other antibodies from binding to the same site, leading to an erroneous interpretation of antigen loss. None of the five patients lacking deep IC antigens had in vivo deposits of $\operatorname{IgG}, \operatorname{IgM}$, or IgA in this location, and in two patients C3 was absent as well. Furthermore, in the cases which could be studied, intercellular deposits of C3 did not block the subsequent binding of IC antibodies to the same sites. IgE and IgD were not looked for, since no abnormal deposits of these classes of immunoglobulin have been reported in pemphigus. Overall, deep intercellular antigens reactive to autologous sera were not expressed in lesions of $33 \%$ of the pemphigus foliaceus patients and in $80 \%$ of those lacking deep intercellular deposits of IgG in vivo.

The causes of antigen loss were not determined. It was more common in lesions than in uninvolved skin of the same patient. While possibly due to tissue damage resulting from the disease, this was unlikely to be the sole explanation, since intercellular antigens were retained in the superficial layers of the epidermis at sites of greatest tissue damage. Furthermore, deep intercellular antigens were absent in the uninvolved skin of at least one patient. The predominant association of antigen loss with skin lesions suggests the intriguing possibility that regional variations in antigen distribution may account for the distinctive distribution of pemphigus lesions.

Chessboard titrations performed by reacting a panel of IC antibodies with a number of skin specimens suggest that there are intercellular antigens of at least two differing specificities in human skin. These can be distinguished by their reactivity with autologous or 
allogeneic IC antibodies and may represent individual and group-specific antigens, respectively. Either or both of these antigens may be lost in pemphigus foliaceus.

The absence of intercellular antigens in the deep layers of the epidermis may in part account for superficial location of lesions in some patients with pemphigus foliaceus. As a consequence, IC antibodies may only be able to react to superficial antigens, a site which corresponds to the anatomical location of lesions.

We have previously reported that another immune mechanism which may account for superficial lesions in pemphigus foliaceus is subcorneal IC antibodies, which react only to antigens in the superficial layers of the epidermis (22). Such antibodies are extremely rare in patients without pemphigus foliaceus, having been found in only $0.1 \%$ of over 1,000 patients with unrelated skin diseases. Subcorneal IC antibodies were present in one patient in this study.

Thus, at least two different mechanisms may account for the location of bullae in the upper layers of the epidermis in pemphigus foliaceus: $(a)$ the presence of IC antibodies directed only to superficial antigens, and $(b)$ the absence of normal deep intercellular antigens. These mechanisms are not mutually exclusive, and either or both may be operative in an individual patient. In both cases, IC antibodies would bind only to the superficial layers of the skin at sites which correspond to, and thus may account for, the superficial locations of lesions in this form of pemphigus.

The ultimate mechanisms producing acantholysis in pemphigus are unknown. Complement activation is suggested by the binding of complement components to the intercellular substance of the epidermis and the presence of reaction products of complement activation in blister fluid of pemphigus lesions $(13,26)$. However, our study indicates that there is often a discrepancy between the site of $\mathrm{C} 3$ deposition, which is predominantly restricted to the deeper layers of the epidermis in pemphigus foliaceus, and that of lesions of the disease, which are always superficial. Furthermore, circulating IC antibodies were unable to fix complement when bound to skin in vitro. These findings, together with the observation that pemphigus serum can cause acantholysis in vitro in the absence of complement (19), suggests that complement activation is a result rather than a cause of the initial injury. Viewed in this light, the predominant location of $\mathrm{C} 3$ in the deep epidermis might be due to diffusion restraints imposed by the large molecular weight of $\mathrm{C} 3$. An alternate mechanism of acantholysis is that binding of intercellular antibodies to the surface of epidermal cells causes activation or release of epidermal proteolytic enzymes (27) or of other factors which in turn lead to acantholysis and activation of complement. Coating of keratinocyte surfaces by intercellular antibodies might also contribute to acantholysis by interfering with the adhesive properties of the cells.

\section{ACKNOWLEDGMENTS}

We wish to thank Mr. Robert Bonomo and Ms. Beverly Smith for their technical assistance.

This research was supported, in part, by funds from the Dermatology Department at New York University School of Medicine and the Chernow Fund.

\section{REFERENCES}

1. Hashimoto, K., and W. F. Lever. 1967. An electron microscopic study of pemphigus vulgaris of the mouth and the skin with special reference to the intercellular cement. J. Invest. Dermatol. 48: 540-552.

2. Wilgram, G. F., J. B. Caulfield, and E. B. Madgic. 1964. An electron microscopic study of acantholysis and dyskeratosis in pemphigus foliaceus. J. Invest. Dermatol. 43: 287-299.

3. Beutner, E. H., and R. E. Jordon. 1964. Demonstration of skin antibodies in sera of pemphigus patients by indirect immunofluorescent staining. Proc. Soc. Exp. Biol. Med. 117: 505-510.

4. Beutner, E. H., W. F. Lever, E. Witebsky, R. E. Jordon, and B. Chertock. 1965. Autoantibodies in pemphigus vulgaris: responses to an intercellular substance of epidermis. J. A. M. A. (J. Am. Med. Assoc.). 192: 682-688.

5. Chorzelski, T. P., J. F. Von Weiss, and W. F. Lever. 1966. Clinical significance of autoantibodies in pemphigus. Arch. Dermatol. 93: 570-576.

6. Thivolet, J., and A. J. Beyvin. 1967. Anticorps antiepiderme dans les dermatoses bulleuses. Documents cliniques et experimentaux. Bull. Soc. Fr. Dermatol. Syphiligr. 74: 300-304.

7. Beutner, E. H., L. S. Pringenzi, W. Hale, C. Leme, and O. G. Bier. 1968. Immunofluorescent studies of autoantibodies to intercellular areas of epithelia in Brazilian pemphigus foliaceus. Proc. Soc. Exp. Biol. Med. 127: 81-86.

8. Burnham, T. K., and G. Fine. 1972. Indirect cutaneous immunofluorescence. Arch. Dermatol. 105: 52-58.

9. Rodriguez, J., and J-C. Bystryn. 1975. The reliability of the indirect immunofluorescent test for pemphigus and bullous pemphigoid. Clin. Res. 23: 454A. (Abstr.)

10. Chorzelski, T. P., and E. H. Beutner. 1969. Factors contributing to occasional failures in demonstration of pemphigus antibodies by the immunofluorescent test. $J$. Invest. Dermatol. 53: 188-191.

11. Beutner, E. H., T. P. Chorzelski, and R. E. Jordon. 1970. Autosensitization in pemphigus and bullous pemphigoid. Charles C. Thomas, Publisher, Springfield, Ill.

12. Jordon, R. E., C. T. Triftshauser, and A. L. Schroeter. 1971. Direct immunofluorescent studies of pemphigus and bullous pemphigoid. Arch. Dermatol. 103: 486-491.

13. Jordon, R. E., A. L. Schroeter, R. S. Rogers, and H. O. Perry. 1974. Classical and alternate pathway activation of complement in pemphigus vulgaris lesions. J. Invest. Dermatol. 63: 256-259.

14. Cormane, R. H., and D. Petzoldt. 1970. Immunofluorescence studies on the affinity of pemphigus antibodies to epithelial intercellular substances. Dermatologica (Basel). 140: 1-8

15. Van Joost, Th., R. H. Cormane, and K. W. Pondman. 1972. Direct immunofluorescent study of the skin on occurrence of complement in pemphigus. Br. J. Dermatol. 87: $466-474$. 
16. Beutner, E. H., R. E. Jordon, and T. P. Chorzelski. 1968. The immunopathology of pemphigus and bullous pemphigoid. J. Invest. Dermatol. 51: 63-80.

17. Peck, S. M., K. E. Osserman, L. B. Weiner, A. Lefkovits, and B. S. Osserman. 1968. Studies in bullous diseases: immunofluorescent serologic tests. N. Engl. J. Med. 279: 951-958.

18. Sams, W. M., Jr., and R. E. Jordon. 1971. Correlation of pemphigoid and pemphigus antibody titres with activity of disease. Br. J. Dermatol. 84: 7-13.

19. Schiltz, J. R., and B. Michel. 1976. Production of epidermal acantholysis in normal human skin in vitro by the IgG fraction from pemphigus serum. J. Invest. Dermatol. 67: 254-260.

20. Inderbitzin, T. M., and P. J. Grob. 1967. Destruction of epithelial cells in vivo by antiepithelial autoantibodies. J. Invest. Dermatol. 49: 642-645.

21. Wood, G. W., E. H. Beutner, and T. P. Chorzelski. 1972. Studies in immunodermatology. II. Production of pemphigus-like lesions by intradermal injection of mon- keys with Brazilian pemphigus foliaceus sera. Int. Arch. Allergy Appl. Immunol. 42: 556-564.

22. Bystryn, J-C., E. Abel, and C. DeFeo. 1974. Pemphigus foliaceus: subcorneal intercellular antibodies of unique specificity. Arch. Dermatol. 110: 857-861.

23. Lever, W. F. 1965. Pemphigus and Pemphigoid. Charles C. Thomas, Publisher, Springfield, Ill.

24. Bystryn, J-C., E. Abel, and A. Weidman. 1973. Antibodies against the cytoplasm of human epidermal cells. Arch. Dermatol. 108: 241-244.

25. Jordon, R. E., W. M. Sams, Jr., and E. H. Beutner. 1969. Complement immunofluorescence staining in bullous pemphigoid. J. Lab. Clin. Med. 74: 548-556.

26. Jordon, R. E., N. K. Day, J. R. Luckasen, and R. A. Good. 1973. Complement activation in pemphigus vulgaris blister fluid. Clin. Exp. Immunol. 15: 53-63.

27. Farb, R. M., E. A. Fountain, F. J. Yost, and G. S. Lazarus. 1977. Molecular model for the induction of acantholysis in pemphigus vulgaris. Clin. Res. 25: 281A. (Abstr.) 